\title{
Biomasa aérea de familias botánicas en un bosque siempreverde piemontano sometido a grados de intervención
}

\section{Aerial biomass of botanical families in piedmont evergreen forest subject to intervention levels}

\author{
Yudel García-Quintana ${ }^{1 *(\mathbb{C})}$, Yasiel Arteaga-Crespo ${ }^{10}$, Bolier Torres-Navarrete ${ }^{1(\mathbb{D})}$, \\ Carlos Bravo-Medina ${ }^{1}$, Marco Robles-Morillo ${ }^{2}$
}

García-Quintana, Y., Arteaga-Crespo, Y., Torres-Navarrete, B., Bravo-Medina, C. y Robles-Morillo, M. (2021). Biomasa aérea de familias botánicas en un bosque siempreverde piemontano sometido a grados de intervención. Colombia Forestal, 24(1), 45-59

Recepción: 14 de febrero 2020

\section{Resumen}

Se evaluó la biomasa aérea acumulada en lo concerniente a familias botánicas en un bosque siempreverde piemontano sometido a diferentes grados de intervención en la zona alta de la microcuenca del río Puyo. En cinco transectos de 0.1 ha se registraron las especies con DAP $\geq 10 \mathrm{~cm}$. Se encontró menor número de familias en sitios con alto grado de perturbación. La biomasa aérea varió de 161.84

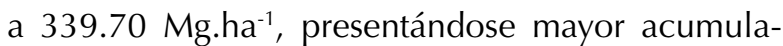
ción en individuos de menor clase diamétrica. Las familias con mayor índice de valor de importancia de biomasa fueron Moraceae, Arecaceae y Euphorbiaceae, reconocidas por su potencial en cuanto a estructura y carbono. El análisis de correspondencia canónica sin tendencia (DCA) indicó variación en la contribución de biomasa para cada sitio, existiendo un pequeño número de familias para mantener las reservas de carbono. Los resultados de este estudio brindan información relevante que facilita la toma
Aprobación: 16 de septiembre 2020

de decisiones con respecto la restauración de sitios perturbados.

Palabras clave: estructura diamétrica, perturbaciones, reservas de carbono, valor de importancia de biomasa.

\begin{abstract}
The accumulated aerial biomass was evaluated at the level of botanical families in an evergreen montane forest subject to different degrees of intervention in the upper Puyo river watershed. In five transects of 0.1 ha, species with diameter at breast height $(\mathrm{DBH}) \geq 10 \mathrm{~cm}$ were recorded. Fewer families were found in sites with a high degree of disturbance. The aerial biomass varied from 161.84 to 339.70 Mg.ha-1, with greater accumulation in individuals of smaller diametric class. The families with the highest biomass importance value index were Moraceae, Arecaceae and Euphorbiaceae, recognized for their potential in terms of structure and carbon.
\end{abstract}

1 Facultad de Ciencias de la Tierra, Universidad Estatal Amazónica (UEA), vía Tena Km 2 1⁄2, Puyo, Pastaza, Ecuador.

2 The Nature Conservancy, Quito, Ecuador.

ygarcia@uea.edu.ec Autor de correspondencia. 
Detrended correspondence analysis (DCA) indicated variation in the contribution of biomass for each site, having a small number of families to maintain carbon stocks. This provides relevant information that facilitates decision making for restoration in disturbed sites.

Key words: diametric structure, disturbances, carbon reserves, importance value of biomass.

\section{INTRODUCCIÓN}

Los bosques coadyuvan a mitigar el cambio climático global mediante el almacenamiento de carbono en la vegetación y en el suelo, así como en el intercambio con la atmósfera. La creciente preocupación y demanda por llevar a cabo estudios de biomasa posibilita comprender el funcionamiento de los bosques y, a su vez, entender cómo los cambios de uso del suelo pueden alterar la estructura, biodiversidad y los servicios ecosistémicos; esto último podría ser revertido o atenuado mediante un adecuado manejo (Krug, 2019; Mori et al., 2017; Tong et al., 2020). Los bosques se destacan por su gran capacidad de fijar carbono en sus estructuras leñosas (Trugman et al., 2018); pues, el fuste de un árbol almacena aproximadamente el $84 \%$ de biomasa, de la cual el $46 \%$ es carbono (Tobías-Baeza et al., 2019). Por ello, cualquier tipo de perturbaciones sobre el ecosistema genera daños ambientales que inciden en el ciclo del carbono (Fu et al., 2017).

La cuenca del Amazonas se constituye como un reservorio con gran diversidad ecológica, pues aglutina el $50 \%$ de los bosques del mundo y desempeña un papel estratégico en el secuestro de carbono (Lathuillière et al., 2016). Los bosques húmedos tropicales tienen una elevada productividad de biomasa aérea y representan una fracción significativa de las existencias totales de reserva de carbono y nutrientes (Jones et al., 2019); pero, aun así, son insuficientes los estudios que permitan profundizar sobre la producción de biomasa aérea (Chave et al., 2005; Torres et al., 2019). El carbono almacenado es un aspecto clave en los ecosistemas de bosques, pues resulta del balance entre la fotosíntesis y la respiración autotrófica; se identifica que parte del $\mathrm{CO}_{2}$ capturado se usa para crear biomasa viva, mientras que el resto se devuelve a la atmósfera. Cuando las hojas o ramas mueren y se descomponen aumenta el carbono del suelo y también se libera una pequeña cantidad a la atmósfera a través de la respiración heterotrófica (Bravo et al., 2017).

Se desconoce el nivel de carbono almacenado en parte de las regiones tropicales, lo que limita la valoración y conservación de estos ecosistemas debido a la escasez de estimaciones de biomasa en vivo y su variación en los diferentes ambientes. Este se distribuye entre tres compartimentos: biomasa de plantas vivas (tallo, ramas, follaje, raíces), detritos de plantas (ramas y conos caídos, basura forestal, tocones de árboles, copas de árboles, troncos) y tierra (humus mineral orgánico, suelo mineral superficial y profundo) (Bravo et al., 2017).

Las estimaciones de biomasa de los bosques tropicales son un medio útil para gestionar la reducción de las emisiones de carbono durante la deforestación y los cambios de uso del suelo, lo que permite aumentar las reservas de carbono, así como mantener los servicios ecosistémicos y la biodiversidad (Messinger et al., 2016). Por esto es importante comprender los procesos que determinan los patrones actuales de almacenamiento de carbono y la estructura para predecir la respuesta de estos bosques a las condiciones ambientales cambiantes.

En la actualidad los estudios de acumulación de biomasa en la Amazonía ecuatoriana son muy importantes para comprender el ciclo del carbono y su papel en los cambios climáticos, pues hay un limitado conocimiento de las familias botánicas y especies arbóreas que más contribuyen a las reservas de carbono. Como se ha dicho, tener disponible este conocimiento incide en el éxito de los programas de restauración al aportar 
información valiosa sobre la captura de carbono como herramienta para mitigar el empobrecimiento de los bosques amazónicos de la región (Yepes et al., 2015).

Con todo lo anterior en cuenta, esta investigación se realizó en la zona alta de la microcuenca del río Puyo, la forma parte de un importante ecosistema del pie de monte amazónico de la provincia de Pastaza entre los 1100 a 1300 m de altitud; considerado como un sitio mega diverso por formar parte del hotspot-Uplands Western Amazonia (Myers et al., 2000). La microcuenca del río Puyo es un escenario donde los seres humanos interactúan con ella y sus recursos naturales; es, además, reconocida por los múltiples beneficios sociales, ambientales y económicos. La importancia de esta área está dada por sus importantes funciones hidroreguladoras, biodiversidad, provisión de alimento, protección del ciclo de nutrientes, retención del carbono, regulación de plagas y polinización. Sin embargo, las actividades antropogénicas y los cambios de uso del suelo han incidido en el deterioro progresivo de gran parte de los ecosistemas de bosques, lo que ha causado serios daños en su cobertura vegetal, cambios en la estructura y composición del suelo producto de la expansión de la frontera agrícola, pérdidas considerables de la biodiversidad, deslizamientos de suelo, disminución de la biomasa acumulada $y$, por consiguiente, afectaciones en los servicios ecosistémicos (Burbano-Orjuela, 2016). Por ello, es importante conocer la contribución de las familias botánicas al stock de carbono y su valor ecológico como herramienta para apoyar los esfuerzos de restauración orientados a la búsqueda de especies y familias claves dentro del ecosistema de bosque objeto de esta investigación. Por lo que el objetivo del presente trabajo fue evaluar la biomasa aérea acumulada por familias botánicas en un bosque siempreverde piemontano sometido a diferentes grados de intervención en la zona alta de la microcuenca del río Puyo, en la Amazonía ecuatoriana.

\section{MATERIALES Y MÉTODOS}

\section{Descripción del área de estudio}

El estudio se realizó en un bosque siempreverde piemontano de la zona alta de la microcuenca del río Puyo, al norte de la cordillera oriental de los Andes, localizado dentro del territorio hidrográfico del río Pastaza donde se encuentra ubicado el sector Pindo Mirador, parroquia Mera, provincia Pastaza, Ecuador (figura 1). Esta zona es poco conocida en la región en cuanto a su composición, estructura y stock de carbono; además, tiene alta relevancia por sus importantes funciones hidroreguladoras. Presenta en promedio 1228.7 $\mathrm{m}$ de altitud, temperatura media anual de $23^{\circ} \mathrm{C}$, precipitación promedio anual de $4119 \mathrm{~mm}$ y una humedad relativa de $84 \%$ (Instituto Nacional de Meteorología en Hidrología del Ecuador, 2014).

En el área de estudio se instalaron cinco transectos permanentes, separados por una distancia de $100 \mathrm{~m}$, con un tamaño de 0.1 ha $(10 \times 100$ $\mathrm{m})$, los cuales fueron sometidos a diferentes niveles de intervención. Las características fisiográficas, grado de perturbación y la composición florística de los sitios se presentan en la tabla 1. Las coordenadas geográficas, altitud y pendiente se obtuvieron con un GPS RTK con precisión de $1 \mathrm{~cm}$, doble frecuencia, 72 canales, GPS + Glonass y receptor- $100 \mathrm{~Hz}$. Los datos de la composición florística fueron obtenidos mediante la fase de inventario, a partir de un muestreo sistemático considerando la accesibilidad y topografía del terreno. El grado de perturbación se evaluó por observación directa en los sitios, a partir de los criterios establecidos por González et al. (2016), con adecuaciones por los autores de esta investigación que corresponden con los disturbios asociados a los factores antrópicos y naturales presentes en el área de estudio. Se consideraron tres grados de perturbación (alta, medio y baja) en función de la presencia de talas, cambios de uso del suelo, pastoreo, extracción de productos 


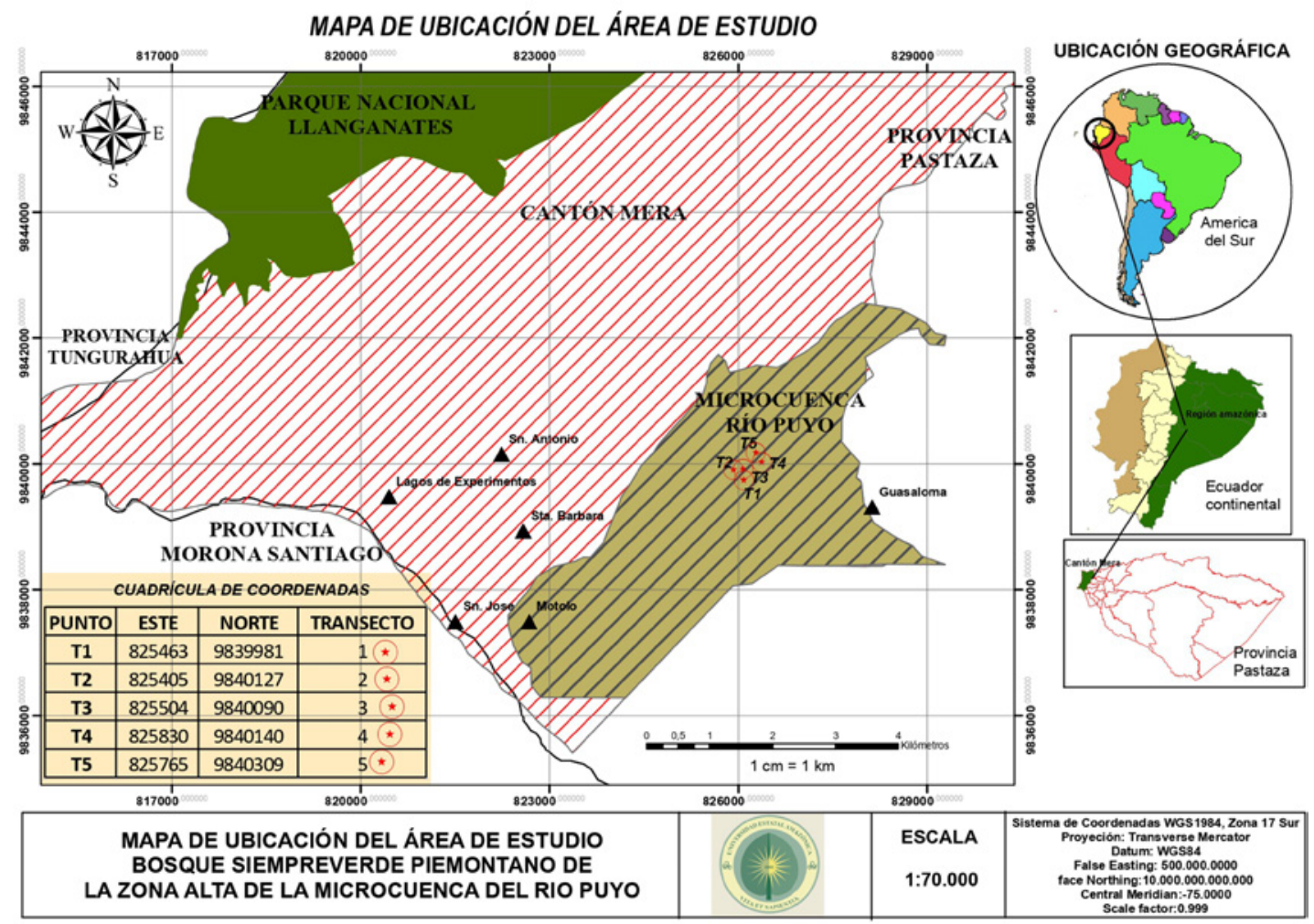

Figura 1. Ubicación geográfica del área de estudio.

forestales no maderables, vegetación secundaria y caída de árboles por efecto del viento o deslizamientos de suelo. Se consideró un grado de perturbación alto cuando la intervención fue severa, con predominio de más de cuatro de los disturbios analizados; medio cuando la intervención fue moderada, determinada por la presencia de tres tipos de disturbios; y bajo cuando la intervención fue escasa como resultado de la presencia de dos o menos disturbios presentes en el sitio.

\section{Inventario florístico}

Se realizó un inventario florístico con todos los individuos con DAP $\geq 10 \mathrm{~cm}$, los cuales fueron identificados preliminarmente en el campo con el apoyo de un experto botánico en flora amazónica. También, se comparó con el libro de los Árboles del Ecuador (Palacios, 2016) y se verificó con la colección de muestras del Herbario Ecuatoriano Amazónico (ECUAMZ) de la Universidad Estatal
Amazónica. El inventario permitió determinar las siguientes características estructurales: número de familias botánicas, área basal, diámetro promedio de los árboles y la riqueza florística, la cual fue calculada mediante el índice de Margalef modificado por Magurran (2013).

\section{Biomasa aérea y valor de importancia}

Se estimó la biomasa aérea por familias (AGB, por sus siglas en inglés), expresada en $\mathrm{Mg} \mathrm{ha}^{-1}$, considerando la contribución individual de cada una de las especies que conforman a la familia botánica independientemente del porte de las especies, por lo que la suma de sus contribuciones comprendió la acumulación de biomasa aérea a nivel de familia. La biomasa aérea se obtuvo a partir de la ecuación alométrica propuesta por Chave et al. (2005), la cual se muestra a continuación:

$\mathrm{AGB}=\rho \times \exp (-1.499+2.148 \ln (\mathrm{DBH})+$ $\left.0.207(\ln (\mathrm{DBH}))^{2}-0.0281(\ln (\mathrm{DBH}))^{3}\right)$ 
Tabla 1. Características fisiográficas y composición florística de los transectos con distintos grados de perturbación

\begin{tabular}{cccccccc}
\hline \multirow{2}{*}{ Transectos } & Coordenadas geográficas & $\begin{array}{c}\text { Altitud } \\
(\mathbf{m s n m})\end{array}$ & $\begin{array}{c}\text { Pendiente } \\
(\mathbf{\%})\end{array}$ & $\begin{array}{c}\text { Número } \\
\text { individuos }\end{array}$ & $\begin{array}{c}\text { Número de } \\
\text { especies }\end{array}$ & $\begin{array}{c}\text { Grado de } \\
\text { perturbaciones }\end{array}$ \\
\cline { 2 - 5 } & $\mathbf{X}$ & $\mathbf{Y}$ & & & & & \\
& 825462 & 9839971 & 1122.4 & 20 & 69 & 23 & Medio \\
& 825488 & 9840077 & 1221.3 & 15 & 52 & 16 & Alto \\
T3 & 825581 & 9840022 & 1234.8 & 10 & 75 & 14 & Bajo \\
T4 & 825822 & 9830132 & 1272.6 & 45 & 60 & 22 & Alto \\
T5 & 825749 & 9840209 & 1292.5 & 30 & 66 & 27 & Medio \\
Total & & & 1228.7 & & 322 & 65 & \\
\hline
\end{tabular}

Se empleó esta ecuación por ser ampliamente utilizada en ecosistemas de bosque siempreverde de la Amazonía (Cuenca et al., 2017; Torres et al., 2019; Vashum y Jayakumar, 2012), la cual presentó una buena aproximación a las condiciones locales para estimar de forma precisa el potencial de biomasa aérea, la cual incluye el diámetro a la altura del pecho ( $\mathrm{DBH}$, por sus siglas en inglés) y la densidad de la madera $(\rho)$. El valor de $(\rho)$ fue posible a partir de los datos reportados de densidad específica de la madera para 23 de las especies inventariadas, con excepción de algunos casos en los que no estuvo disponible y se empleó la $(\rho)$ media global para América del Sur tropical que fue de 0.632 g.cm ${ }^{-3}$ (Chave et al., 2009).

La biomasa acumulada en $\mathrm{Mg}_{\text {g. }} \mathrm{ha}^{-1}$ se calculó por clases diamétricas (CD), las cuales se agruparon en intervalos de $10 \mathrm{~cm}$; siendo la clase inferior $10-20 \mathrm{~cm}$ y la superior con $40 \mathrm{~cm}$ o más. Se determinó el índice de valor de importancia de biomasa a nivel de familias botánicas (BIVF, por sus siglas en inglés) a partir de la metodología propuesta por Torres et al. (2019), con adecuaciones por los autores de este trabajo, la cual se relaciona con la densidad relativa (NRF), área basal relativa (BAF) y biomasa relativa por encima del suelo (AGBF) a nivel de familias botánicas. El BIVF se calculó de la siguiente manera:

$\mathrm{BIVF}=(\mathrm{NRF}+\mathrm{BAF}+\mathrm{AGBF}) / 3$

\section{Procesamiento de datos}

Los datos fueron procesados mediante un análisis de correspondencia canónica sin tendencia (DCA) con el uso del programa ecológico Canoco ver.5.0 (Ter Braak y Smilauer, 2012). Se empleó el DCA de acuerdo con lo expuesto por Ter Braak (1986), pues el valor del gradiente resultó con 4.3 unidades, por lo que el método lineal no fue apropiado. La matriz de datos se elaboró con las unidades de muestreo (cinco sitios de estudio) y los valores de la biomasa acumulada por familias botánicas, a los cuales se le realizó una transformación logarítmica $y^{\prime}=\log (y+1)$. Este análisis permitió comprobar la correlación existente entre las unidades de muestreo y la biomasa acumulada por familias botánicas, además de entender la contribución de las familias botánicas para mantener las reservas de carbono en cada uno de los sitios de estudio. Este análisis aporta información valiosa que facilita la selección de familias claves para cada hábitat desde el punto de vista de su estructura ecológica y aporte de biomasa.

\section{RESULTADOS}

El inventario florístico en 0.5 ha (cinco transectos) reportó un total de 30 familias botánicas, 65 
especies y 322 individuos con DAP $\geq 10 \mathrm{~cm}$; lo que da como resultado un ecosistema con una alta riqueza de familias, pero con baja abundancia de especies. Las familias que presentaron mayor número de especies fueron: Fabaceae, Euphorbiaceae y Lauraceae con 15, 10 y 8 taxones, respectivamente, que representaron un $50.77 \%$ del total de especies inventariadas en la zona de estudio. Siguen, en orden de representación, tres familias (Urticaceae, Arecaceae y Melastomataceae) con siete especies; luego, tres familias (Annonaceae, Burseraceae, Malvaceae) con una composición de cuatro y seis especies; posteriormente, siete familias (Asterecaeae, Clusiaseae, Meliaceae, Moraceae, Rosaseae, Rubiaceae y Salicaceae) con dos y tres especies; y, por último, 14 familias con una sola especie (figura 2).

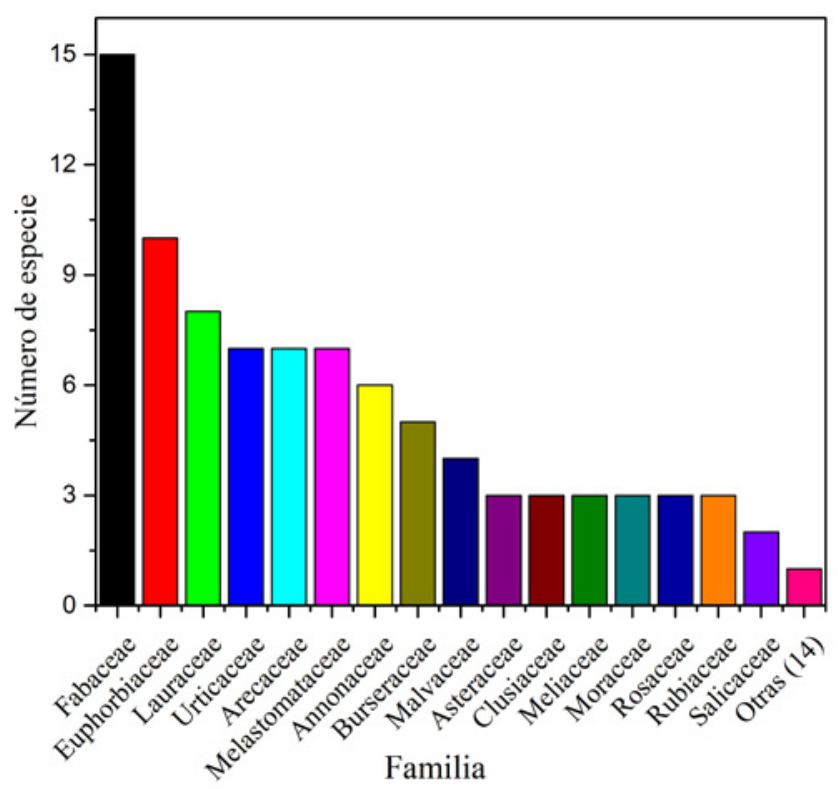

Figura 2. Composición florística del bosque siempreverde piemontano de la zona alta, microcuenca río Puyo.

Las características estructurales del bosque resultaron variables para cada uno de los sitios con diferentes grados de perturbación (tabla 2). Se encontró que los sitios y T4 sometidos a alto grado de perturbación, fundamentalmente por factores antrópicos, presentaron características estructurales determinadas por menor número de familias botánicas por hectárea, área basal por hectárea y diámetro promedio, mientras que la riqueza florística fue superior. Esto pudiera deberse a la relación proporcional que existió entre el número de especies y número de individuos, siendo los sitios que presentaron menor composición florística. Se reportó la presencia de solo cuatro familias presentes en todos los sitios del total del inventario, lo que sugiere que incluso en una pequeña área la diversidad de familias puede variar.

Tabla 2. Características estructurales del bosque siempreverde piemontano por transectos

\begin{tabular}{|c|c|c|c|c|c|}
\hline \multirow{2}{*}{ Variables } & \multicolumn{5}{|c|}{ Transecto } \\
\hline & & & T3 & T4 & T5 \\
\hline $\begin{array}{l}\text { Número de } \\
\text { familias }\end{array}$ & 15 & 11 & 12 & 11 & 19 \\
\hline $\begin{array}{l}\text { Riqueza } \\
\text { florística }\end{array}$ & 34.8 & 37.3 & 34.13 & 36 & 37.17 \\
\hline Área basal $\left(\mathrm{cm}^{2}\right)$ & 40.77 & 29.61 & 34.88 & 30.15 & 33.36 \\
\hline $\begin{array}{c}\text { Diámetro } \\
\text { promedio }(\mathrm{cm})\end{array}$ & 25.96 & 18.86 & 22.22 & 19.21 & 21.22 \\
\hline
\end{tabular}

La biomasa aérea acumulada (AGB) en cada uno de los transectos de estudio osciló en un rango de 161.84 a 339.70 Mg.ha-1. Estos resultados mostraron variaciones en la biomasa total, reportándose una mayor acumulación en los sitios y T5 con valores de 348.53 y 339.70 Mg.ha- $^{-1}$ y menores en y T4 con 169.03 y 161.84 Mg.ha- $^{-1}$, respectivamente.

La mayor acumulación de biomasa aérea se presentó en las clases diamétricas superiores $(C D>40 \mathrm{~cm}$ ) y la menor en la inferior (CD 10-20 $\mathrm{cm})$; siendo la contribución de la biomasa acumulada diferente en cada uno de los sitios de estudio y clases diamétricas, con menor variación entre los sitios en la clase inferior (figura 3). En la clase diamétrica inferior se concentró la mayor cantidad de individuos, mientras que hubo escasa representación de árboles con diámetros mayores a $40 \mathrm{~cm}$. Esto sugiere que la distribución de las especies y 
familias en cada transecto no es uniforme y pudiera estar determinada por los diferentes grados de perturbaciones predominante en la zona, debido a los fuertes vientos y deslizamientos de suelo que causan mortalidad de los árboles más grandes, lo cual se puede manifestar en la capacidad de almacenamiento de carbono.

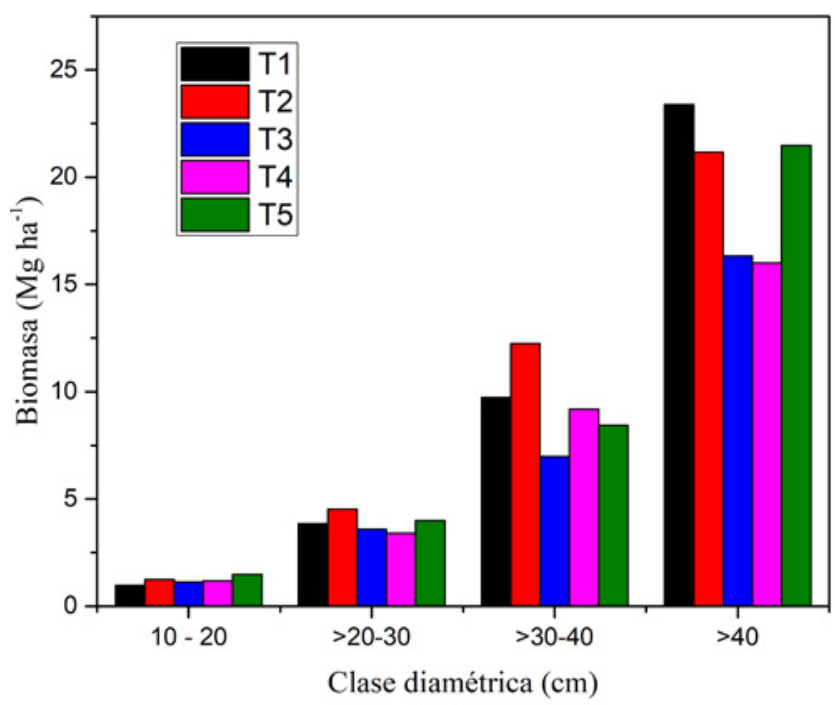

Figura 3. Biomasa aérea acumulada por clases diamétricas en los transectos de estudio.

El BIVF calculado en función de la densidad, área basal y biomasa aérea relativa resultó ser un buen indicador para identificar las familias botánicas potenciales en la captura de carbono y estructura del bosque. Los resultados del BIVF (tabla 3), a partir de la contribución individual de cada una de las especies (independientemente de su hábito o porte), evidenció que las familias pueden ser de importancia relativa para estructura, pero no para almacenamiento de carbono o viceversa. Las tres familias con mayor BIVF fueron: Moraceae, Arecaceae, Euphorbiaceae y después le siguen Asteraceae, Fabaceae y Rubiaceae, lo que indica que las más abundantes no son necesariamente esenciales para biomasa o carbono. Las representadas en los cinco transectos de estudio fueron: Euphorbiaceae, Arecaceae, Fabaceae y Melastomataceae, debido a su alta abundancia. Las de mayor contribución a la biomasa fueron: Moraceae, Rubiaceae y Rutaceae. Esto demuestra la contribución de un pequeño número de familias para mantener el nivel de reservas de carbono. Este patrón de baja representatividad de familias en cuanto a carbono podría indicar que los futuros cambios del ecosistema pueden resultar en el empobrecimiento de este importante reservorio del bosque amazónico.

El DCA describió la distribución de la biomasa acumulada en función de los sitios de estudio y tuvo como resultado una alta correlación entre los transectos y la biomasa acumulada. El primer eje fue capaz de explicar el $74 \%$ y el segundo eje el $21 \%$ de la varianza total explicada. Se comprobó mediante el análisis de ordenación espacial que el sitio T4 fue el más distante, determinado por el ángulo que forma aproximado de $90^{\circ}$ en relación con los demás (figura 4), resultando el de menor aporte de biomasa. En cambio, los sitios y T5 estuvieron distribuidos muy cerca, siendo los de mayor abundancia de familias y a la vez con mayor contribución de biomasa. Estos resultados sugieren que existe variación en la contribución de biomasa por familias para cada uno de los sitios.

Las familias que más contribuyeron a la biomasa en el sitio fueron seis (Malvaceae, Rubiaceae, Sapindaceae, Lamiaceae, Cyatheaceae y Moraceae); en se agruparon cuatro familias (Fabaceae, Burseraceae, Melastomataceae y Lauraceae) con menor cantidad de familias contribuyentes al total de biomasa acumulada; T3 resultó con cinco familias (Annonaceae, Rosaceae, Myrtaceae, Rutaceae y Protaceae); T4 estuvo representado por cinco familias (Meliaceae, Boraginaceae, Elaeocarpaceae, Siparunaceae y Arecaceae); y T5 con ocho familias participantes (Sapotaceae, Saliaceae, Araliaceae, Urticaceae, Phyllantaceae, Lecythidaceae, Asteraceae y Celastraceae), resultando con mayor número de familias. Se evidenció que las familias Clusiaceae y Euphorbiaceae mostraron un patrón diferente a las demás; las cuales no estuvieron asociadas a ninguno de los transectos, lo que indica una menor correlación con el resto de la composición familiar. Esta característica se debió a la baja representatividad como reservas de carbono. 


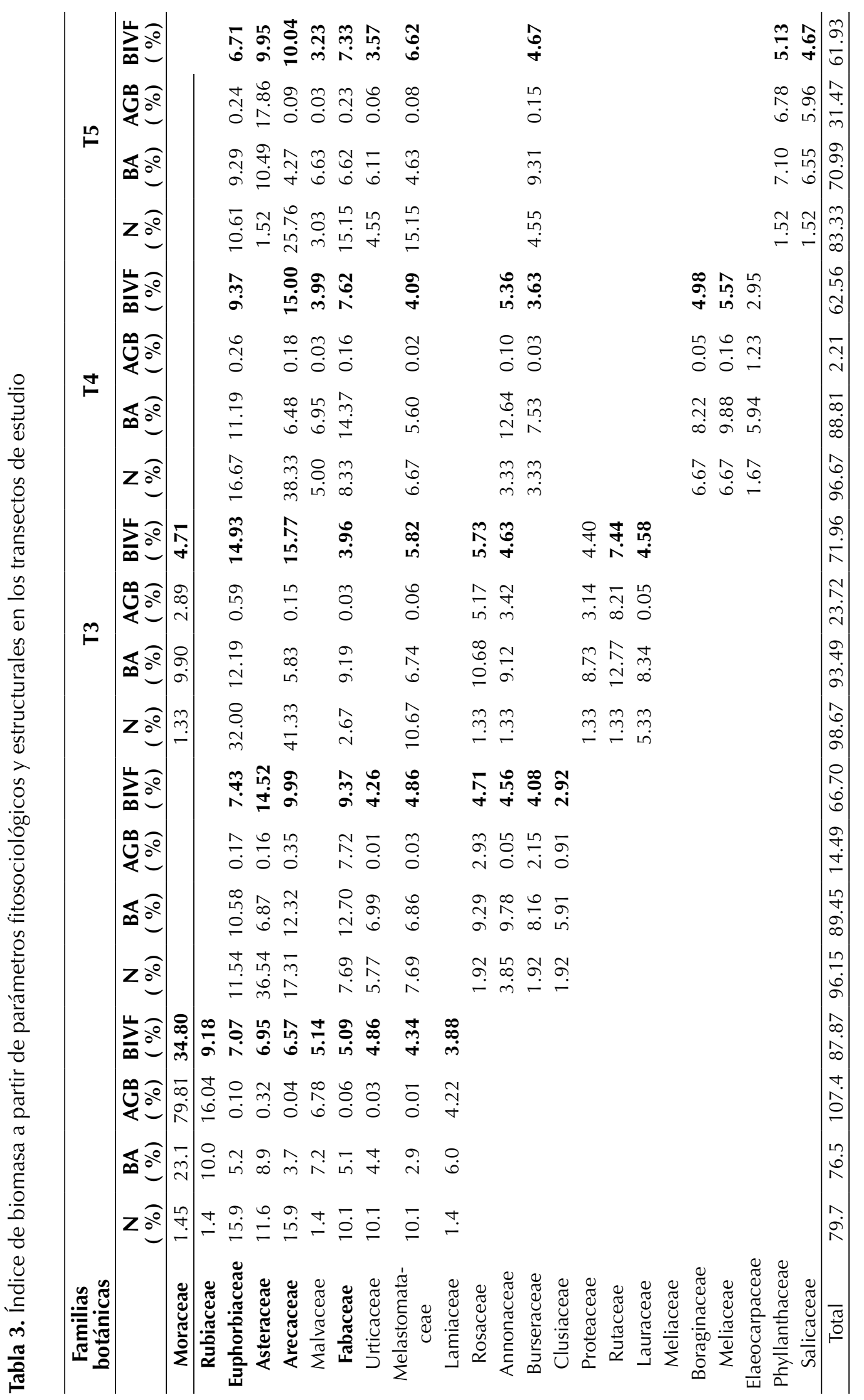


Los resultados obtenidos posibilitaron la identificación de las familias botánicas que más contribuyeron a la reserva de carbono en cada sitio y, a su vez, las que más aportan a la estructura del ecosistema de bosque siempreverde piemontano de la zona alta de la microcuenca del río Puyo. Existe un pequeño número de familias con garantía de mantener las reservas de carbono. Esto las convierte en familias claves desde el punto de vista funcional en estos hábitats y deben ser consideradas como prioritarias para futuras acciones de restauración.

\section{DISCUSIÓN}

La alta riqueza y composición de familias botánicas, así como la distribución desigual en cuanto al número de especies, se corresponde con los patrones florísticos descritos para la Amazonía (Cabrera-Amaya y Rivera-Diaz, 2016; Jadán et al., 2017). Se puede apreciar que se comparten familias con gran riqueza de especies como Fabaceae, Euphorbiaceae, Lauraceae, Urticaceae, Arecaceae, Melastomataceae y Annonaceae, reconocidas como algunas de las más importantes en el neotrópico (Gentry, 1982);

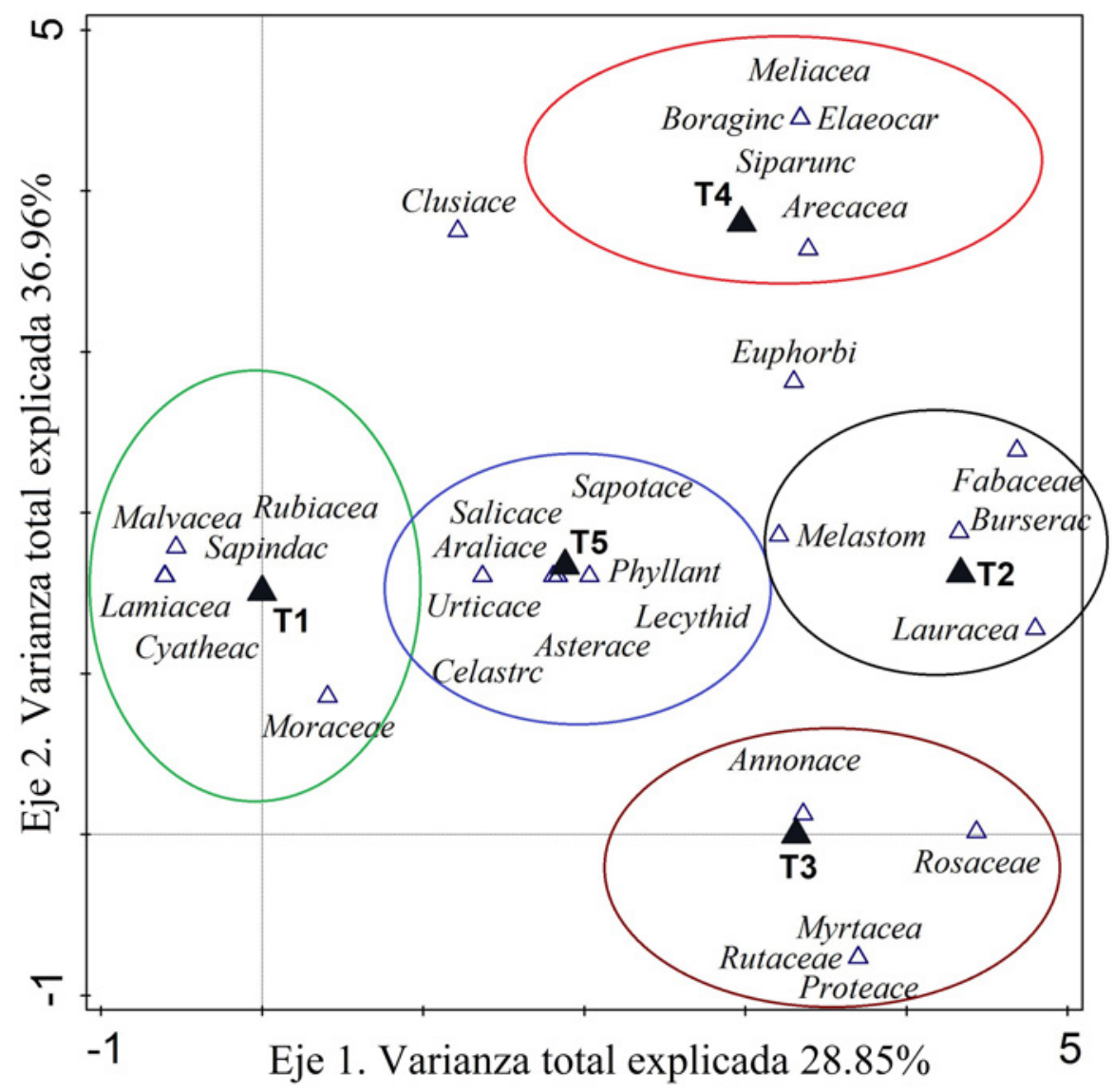

Figura 4. Ordenación espacial de los transectos y acumulación de biomasa aérea por familias botánicas.

Leyenda: Meliaceae (Meliacea); Boraginaceae (Boraginc); Elaeocarpaceae (Elaeocar); Siparunaceae (Siparunc); Clusiaceae (Clusiace); Arecaceae (Arecacea); Euphorbiaceae (Euphorbi); Malvaceae (Malvacea); Rubiaceae (Rubiacea); Salicaceae (Salicace); Sapotaceae (Sapotace); Fabaceae (Fabaceae); Sapindaceae (Sapindac); Araliaceae (Araliace); Melastomataceae (Melastom); Burseraceae (Burserac); Lamiaceae (Lamiacea); Urticaceae (Urticace); Phyllanthaceae (Phyllant); Lecythidaceae (Lecythid); Lauraceae (Lauracea); Cyatheaceae (Cyatheac); Asteraceae (Asterace); Celastraceae (Celastrc); Moraceae (Moraceae); Annonaceae (Annonace); Rosaceae (Rosaceae); Myrtaceae (Myrtacea); Rutaceae (Rutaceae); Proteaceae (Proteace). 
además de constituir elementos distintivos del bosque húmedo tropical caracterizados por una flora entre la vegetación de tierras bajas y los de cordillera (Ter Steege et al., 2013). Sin embargo, lo más consistente es el patrón que se repite en los bosques siempreverde piemontano de la microcuenca del río Puyo con la familia Fabaceae que resultó en el primer lugar según la riqueza de especies, catalogada como una de las más ricas en bosques de tierra baja en el neotrópico (Gentry, 1988). Por otro lado, Ilama la atención la ubicación de las familias Arecaceae, Rubiaceae y Moraceae que han sido reportadas con mayor riqueza para las condiciones amazónicas, no existiendo coincidencias en cuanto a su representatividad en el área de estudio (Cabrera-Amaya y Rivera-Diaz, 2016; Flores-Valencia et al., 2016; Torres et al., 2019).

En un bosque siempreverde piemontano primario del norte de la cordillera oriental de los Andes, entre los 600-700 m de altitud, en las proximidades del río Piatúa que tributa al río Anzu, cantón Arosemena Tola, provincia de Napo de la Amazonía ecuatoriana, Patiño et al. (2015) reportaron la familia Arecaceae con el mayor porcentaje de especies (22.92\%), seguido de Lauraceae con un $15.97 \%$ y en tercer lugar la familia Vochysiaceae con $6.60 \%$. Esto indica que solo la familia Lauraceae ha sido altamente representada en el área de estudio. Ello sugiere que los diferentes grados de perturbación observados en los sitios pueden haber influido en la variación de la composición florística asociada al aumento de la deforestación y fragmentación que resulta en la pérdida de hábitat de especies de flora (Quintana et al., 2017), así como la extracción de madera (Vasco et al., 2017).

El alto nivel de heterogeneidad encontrada en los parámetros de estructura y composición florística del bosque siempreverde piemontano de la zona alta de la microcuenca del río Puyo, determinado por un menor número de familias botánicas, área basal, diámetro promedio y mayor riqueza florística en los sitios más perturbados (T2 y T4), demostró que la gravedad de las perturbaciones que se presentan con frecuencia en esta importante zona de protección altera los patrones ecológicos de este piedemonte amazónico. En este escenario es necesario promover acciones de restauración que permitan la rehabilitación de los sitios más perturbados a partir de grupos núcleos de familias botánicas para cada condición local. Estos resultados se corresponden con lo descrito por Valdés-Sáenz et al. (2014), Gutiérrez y Becerra (2018) y Vega (2020), quienes refieren que las perturbaciones antrópicas alteran sensiblemente los patrones de estructura y composición en las comunidades vegetales.

Además de estos criterios, las diferencias contrastantes en riqueza, estructura y composición florística podrían explicarse por la heterogeneidad ambiental que existe, determinada fundamentalmente por las condiciones topográficas (pendiente y altitud) (Cueva et al., 2019) que propician la caída de muchos árboles y, por consiguiente, favorece las trayectorias sucesionales del bosque.

Los resultados obtenidos en cuanto al contenido de biomasa aérea en la zona alta del bosque siempreverde piemontano de la microcuenca del río Puyo en la Amazonía ecuatoriana son similares a lo reportado por Torres et al. (2019) en un bosque siempreverde de Napo con valores de 290 a 340 Mg.ha-1 ${ }^{-1}$ También concuerdan con lo obtenido en un bosque de tierra firme de la Amazonía colombiana en el que se estimó una biomasa para individuos con DAP $\geq 10 \mathrm{~cm}$ de 297.6 Mg.ha-1 (Paky et al., 2017). La biomasa reportada en el presente estudio se encuentra dentro de lo reportado para la cuenca amazónica (Paky et al., 2017). En estudios de bosques de Amazonía oriental central se obtuvo un valor de $316.8 \pm 79.7$ y en Amazonía occidental $239.8 \pm 58$, valores dentro de los rangos de la presente investigación (Mitchard et al., 2014). Sin embargo, los valores obtenidos en los sitios T2 y T4 fueron relativamente bajos en comparación con el resto de los sitios de estudio y los datos reportados por los autores mencionados. Los valores bajos de biomasa se pueden atribuir al alto 
grado de perturbación reportado en estos sitios por actividades como la extracción de madera, deslizamientos de suelo y fuertes vientos (tabla 1) que propiciaron la caída de muchos árboles y, por consiguiente, una disminución de la biomasa aérea sobre el suelo.

Los valores de biomasa aérea por clases diamétricas se encontraron en los rangos reportados por otros investigadores en bosques amazónicos (Quiceno et al., 2016; Torres et al., 2019; Yepes et al., 2015). De acuerdo con el análisis realizado por Keeling y Phillips (2007), los bosques tropicales del mundo por lo general no tienen valores de biomasa por encima de los $350 \mathrm{Mg}$.ha-1. Ureta (2015) menciona que los bosques amazónicos son un gran almacén de biodiversidad y funcionan como sumideros de carbono debido a la biomasa aérea que acumulan en las diversas especies arbóreas. La mayor concentración de individuos en clases diamétricas pequeñas es consistente con las características de bosque secundario (Cabrera-Amaya y Rivera-Diaz, 2016), lo que da cuenta del efecto de los disturbios en la zona de estudio.

El BIVF, relacionado con el valor de importancia tradicional calculado comúnmente con base en los parámetros fitosociológicos de abundancia, frecuencia y dominancia permitió el análisis ecológico de las comunidades vegetales orientado a interpretaciones de la importancia relativa de las familias botánicas (Torres et al., 2019). Ello proporciona información cuantitativa sobre la acumulación de biomasa, producción y calidad del sitio o condición ambiental de cada hábitat en específico ocupado por grupos de familias botánicas. Lo que ayuda a fundamentar sobre bases científicas las familias de mayor valor ecológico y capacidad para mantener las reservas de carbono. Esto facilita la toma decisiones en los programas de restauración como contribución a los servicios ecosistémicos relacionados con las funciones de regulación y protección del ecosistema de pie de monte amazónico. En un estudio sobre estructura y biomasa a lo largo de un gradiente altitudinal en el bosque siempreverde piemontano de Napo en Ecuador, Torres et al. (2019) obtuvieron resultados similares en cuanto al índice de biomasa, pero a nivel de especies, identificando las que más contribuyen a la biomasa y estructura.

Las afectaciones que sufren los bosques siempreverde piemontano de la zona alta de la microcuenca del río Puyo, determinadas por sitios que poseen una baja abundancia de familias botánicas como contribuyentes a la biomasa aérea acumulada, pueden resultar en el empobrecimiento de la captura de carbono de este importante reservorio de bosque. Vargas et al. (2019) refieren que la capacidad de los ecosistemas para almacenar carbono en forma de biomasa varía en función de la composición florística, la edad y densidad de la población. Esto se corresponde con los resultados del presente estudio, reportando variaciones en los sitios en cuanto a la composición florística, con estadios sucesiones que propician una masa vegetal disetánea y distinta densidad arbórea. Ello es de resaltar pues, según Ureta (2015), los bosques tropicales han captado un gran interés debido a que contribuyen en la captura de $\mathrm{CO}_{2}$ en función de la biomasa aérea proporcionada por innumerables especies vegetales y las alteraciones en ellos pueden influir fuertemente en las emisiones y la fijación de carbono.

El patrón encontrado de baja representatividad de familias botánicas que acumulan carbono podría indicar que los futuros cambios del ecosistema pueden resultar en el empobrecimiento de los bosques amazónicos. Estos resultados se corresponden con lo reportado por Fauset et al. (2015) para los bosques amazónicos donde refieren que la producción de biomasa está altamente concentrada en pocas especies y que pueden ser menos resistentes a cambios ambientales de lo que se esperaría, dado que la alta diversidad de especies por lo general confiere una alta resiliencia ecológica. Del mismo modo, esta información proporciona una mejor comprensión de cómo las existencias de carbono en ecosistemas de bosques tropicales 
podrían conducir a predicciones sobre los cambios de uso de suelos y futuros escenarios de cambio climático. En la zona alta de la microcuenca del río Puyo se constató, mediante el análisis del grado de perturbaciones, que son muy frecuentes las prácticas de cambio de uso del suelo, lo cual propicia alteraciones en la biomasa acumulada del bosque siempreverde piemontano. Estos elementos facilitan información básica para entender los stocks de biomasa en cada uno de los sitios de estudio.

Los resultados obtenidos del análisis de distribución espacial permitieron determinar las tendencias de cada uno de los sitios en cuanto a la acumulación de biomasa por familias botánicas. El comportamiento espacial encontrado obedece al grado de perturbaciones predominante en cada sitio. El análisis global de la distribución de especies y la composición dentro de un mosaico de paisaje permite entender el aporte y el papel que tienen cada uno de los hábitats disponibles en la distribución de las especies y la capacidad para garantizar su conservación en paisajes intervenidos (Petit y Petit, 2003).

De lo anterior se puede concluir que conocer la variación espacial local es importante para predecir el almacenamiento de carbono en los bosques de la Amazonía ecuatoriana. Los estudios que integran etapas sucesionales, topografía y otras fuentes de heterogeneidad local influyen en la estimación de la biomasa (Chave et al., 2003; de Castilho et al., 2006). Los sitios de estudio están caracterizados por estos elementos, lo que permite que se presenten diferentes tendencias de distribución en función de la acumulación de biomasa.

\section{CONCLUSIONES}

La composición florística y estructural del bosque siempreverde piemontano de la zona alta de la microcuenca del río Puyo fue variable para los sitios de estudio sometidos a distintos niveles de perturbación. Esto se reflejó en el potencial de biomasa aérea acumulada que resultó con valores

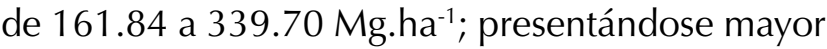
stock de carbono en los sitios y T5 y en la menor clase diamétrica.

El BIVF permitió reconocer familias claves para la captura de carbono y estructura ecológica del bosque (Moraceae, Arecaceae y Euphorbiaceae), demostrándose una variación en la contribución de familias para mantener el nivel de reservas de carbono. Por lo que se apunta a que las prácticas de aprovechamiento y cambio de uso de suelo generarían un alto riesgo en los árboles de mayor potencial de almacenamiento de carbono y pueden propiciar el empobrecimiento de las reservas de carbono en el bosque siempreverde piemontano de la zona alta de la microcuenca del río Puyo.

EI DCA reportó la distribución de las familias en cuanto a reservas de carbono por cada uno de los sitios de estudio, identificando de forma tendencial las que más aportan a la reserva de carbono, a nivel local, con predominio de un pequeño número. Esto brinda información importante que facilita la selección de grupos de familias para la toma de decisiones relacionadas con la restauración de los sitios más perturbados de este reservorio de bosque.

\section{AGRADECIMIENTOS}

Los autores agradecen a las instituciones públicas y a las no gubernamentales que apoyaron con la logística, personal de campo y académicos que posibilitaron el realizar el trabajo de inventario en la zona alta de la microcuenca del río Puyo. Tal es el caso de la Universidad Estatal Amazónica, el Gobierno Autónomo Descentralizado Provincial de Pastaza y la ONG The Nature Conservancy.

\section{CONFLICTO DE INTERESES}

Los autores declaran que no existen conflictos de intereses. 


\section{CONTRIBUCIÓN POR AUTOR}

Todos los autores contribuyeron de manera equitativa y son los únicos responsables de la obra en todos los aspectos que condujeron a la elaboración de su publicación.

\section{REFERENCIAS}

Bravo, F. O., del Río, M., Bravo-Oviedo, A., Montero, G., Ruiz Peinado, R. y del Peso, C. (2017). Forest carbon sequestration: the impact of forest management. En F. Bravo, V. Lemay y R. Jandl, Managing Forest Ecosystems: The Challenge of Climate Change (pp. 251-275). https://doi.org/10.1007/978-3-319-28250-3_13

Burbano-Orjuela, H. (2016). El suelo y su relación con los servicios ecosistémicos y la seguridad alimentaria. Revista de Ciencias Agrícolas, 33(2), 117-124. http://doi.org/10.22267/rcia.163302.58

Cabrera-Amaya, D. y Rivera-Diaz, O. (2016). Floristic composition and structure of riparian forests of the lower basin of Pauto River, Casanare, Colombia. Caldasia, 38(1), 53-85. http://doi.org/10.15446/caldasia.v38n1.57829

Chave, J., Andalo, C., Brown, S., Cairns, M. A., Chambers, J. Q., Eamus, D., Fölster, H., Fromard, F., Higuchi, N. y Kira, T. (2005). Tree allometry and improved estimation of carbon stocks and balance in tropical forests. Oecologia, 145(1), 87-99. https://doi.org/10.1007/s00442-005-0100-x

Chave, J., Condit, R., Lao, S., Caspersen, J. P., Foster, R. B. y Hubbell, S. P. (2003). Spatial and temporal variation of biomass in a tropical forest: results from a large census plot in Panama. Journal of Ecology, 91(2), 240-252. https://doi.org/10.1046/j.1365-2745.2003.00757.x

Chave, J., Coomes, D., Jansen, S., Lewis, S. L., Swenson, N. G. y Zanne, A. E. (2009). Towards a worldwide wood economics spectrum. Ecology Lettersm, 12(4), 351-366. https://doi.org/10.1111/j.1461-0248.2009.01285.x
Cuenca, M.E., Jadán, O., Cueva, K. y Aguirre, C. (2017). Carbono y ecuaciones alométricas para grupos de especies y bosque de tierras bajas, Amazonía Ecuatoriana. Cedamaz, 4(1). http://192.188.49.30/index.php/cedamaz/article/ view/226

Cueva, E., Lozano, D. y Yaguana, C. (2019). Efecto de la gradiente altitudinal sobre la composición florística, estructura y biomasa arbórea del bosque seco andino, Loja, Ecuador. Bosque (Valdivia), 40(3), 365-378. http://doi.org/10.4067/S0717-92002019000300365

de Castilho, C. V., Magnusson, W. E., de Araújo, R. N. O., Luizao, R. C., Luizao, F. J., Lima, A. P. y Higuchi, N. (2006). Variation in aboveground tree live biomass in a central Amazonian Forest: Effects of soil and topography. Forest Ecology and Management, 234(1-3), 85-96. https://doi.org/10.1016/j.foreco.2006.06.024

Fauset, S., Johnson, M. O., Gloor, M., Baker, T. R., Monteagudo, A., Brienen, R. J., Feldpausch, T. R., Lopez-Gonzalez, G., Malhi, Y. y Ter Steege, H. (2015). Hyperdominance in Amazonian forest carbon cycling. Nature Communications, 6(1), 1-9. https://doi.org/10.1038/ncomms7857

Flores-Valencia, M., Araujo-Murakami, A., Cabrera-Severich, P. G., Zuck, D. C., Molina-Olivera, A. y Lazarte-Chispa, M. (2016). Diversidad y composición florística de los bosques amazónicos del sur de la amazonia en el sector Kenia, Guarayos, Bolivia. Kempffiana, 12(2), 20-46.

Fu, Z., Li, D., Hararuk, O., Schwalm, C., Luo, Y., Yan, L. y Niu, S. (2017). Recovery time and state change of terrestrial carbon cycle after disturbance. Environmental Research Letters, 12(10), 104004. https://doi.org/10.1088/1748-9326/aa8a5c

Gentry, A. H. (1982). Neotropical floristic diversity: phytogeographical connections between Central and South America, Pleistocene climatic fluctuations, or an accident of the Andean orogeny? Annals of the Missouri Botanical Garden, 69(3), 557-593.

Gentry, A. H. (1988). Changes in plant community diversity and floristic composition on environmental and geographical gradients. Annals of the Missouri Botanical Garden, 75(1), 1-34. 
González, A. J., Andrade, G. A. P., Sospedra, R. S. y Rodríguez, M. P. R. (2016). Perturbaciones humanas sobre la composición y estructura del bosque semideciduo mesófilo, reserva de la biósfera Sierra del Rosario, Cuba. Sathiri, 10, 196-206.

Gutiérrez, I. y Becerra, P. (2018). Composición, diversidad y estructura de la vegetación de bosques ribereños en el centro sur de Chile. Bosque (Valdivia), 39(2), 239-253. http://doi.org/10.4067/S0717-92002018000200239

Instituto Nacional de Meteorología en Hidrología del Ecuador (2014). Anuario meteorológico del Ecuador. Instituto Nacional de Meteorología en Hidrología del Ecuador.

Jadán, O., Quizhpe, W., Pacheco, E., Aguirre, Z., González, M., Ponce, E. y Peña, D. (2017). Riqueza florística y carbono almacenado en tres pisos altitudinales de bosques amazónicos, Zamora Chinchipe, Ecuador. Bosques Latitud Cero, 7(1).

Jones, I. L., DeWalt, S. J., Lopez, O. R., Bunnefeld, L., Pattison, Z. y Dent, D. H. (2019). Above-and belowground carbon stocks are decoupled in secondary tropical forests and are positively related to forest age and soil nutrients respectively. Science of The Total Environment, 697, 133987. https://doi.org/10.1016/j.scitotenv.2019.133987

Keeling, H. C. y Phillips, O. L. (2007). The global relationship between forest productivity and biomass. Global Ecology and Biogeography, 16(5), 618-631. https://doi.org/10.1111/j.1466-8238.2007.00314.x

Krug, J. H. (2019). How can forest management increase biomass accumulation and $\mathrm{CO} 2$ sequestration? A case study on beech forests in Hesse, Germany. Carbon Balance and Management, 14(1), 17. https://doi.org/10.1186/s13021-019-0132-x

Lathuillière, M. J., Coe, M. T. y Johnson, M. S. (2016). A review of green-and blue-water resources and their trade-offs for future agricultural production in the Amazon Basin: what could irrigated agriculture mean for Amazonia? Hydrology and Earth System Sciences, 20(6), 2179. https://doi.org/10.5194/hess-20-2179-2016

Magurran, A. E. (2013). Measuring biological diversity. John Wiley \& Sons.
Messinger, M., Asner, G. P. y Silman, M. (2016). Rapid assessments of Amazon forest structure and biomass using small unmanned aerial systems. Remote Sensing, 8(8), 615. https://doi.org/10.3390/rs8080615

Mitchard, E. T., Feldpausch, T. R., Brienen, R. J., Lopez-Gonzalez, G., Monteagudo, A., Baker, T. R., Lewis, S. L., Lloyd, J., Quesada, C. A. y Gloor, M. (2014). Markedly divergent estimates of A mazon forest carbon density from ground plots and satellites. Global Ecology and Biogeography, 23(8), 935-946. https://doi.org/10.1111/geb.12168

Mori, A. S., Lertzman, K. P. y Gustafsson, L. (2017). Biodiversity and ecosystem services in forest ecosystems: a research agenda for applied forest ecology. Journal of Applied Ecology, 54(1), 12-27. https://doi.org/10.1111/1365-2664.12669

Myers, N., Mittermeier, R. A., Mittermeier, C. G., Da Fonseca, G. A. y Kent, J. (2000). Biodiversity hotspots for conservation priorities. Nature, 403(6772), 853. https://doi.org/10.1038/35002501

Paky, E., Moreno, F. y Álvarez-Dávila, E. (2017). Contenido de carbono en un bosque de tierra firme del resguardo Nonuya-Villazul, amazonia colombiana. Colombia Forestal, 20(2), 144-157. http://doi.org/10.14483/udistrital.jour.colomb. for.2017.2.a04

Palacios, W. (2016). Árboles del Ecuador: especies representativas. Universidad Técnica del Norte.

Patiño, J., Lozano, P., Tipán, C., Navarrete, H., López, R., Asanza, M. y Torres, B. (2015). Composición florística y estructura de un bosque siempreverde piemontano de 600 a $700 \mathrm{~m}$ snm en la cuenca del río Piatúa, Napo, Ecuador. Revista Amazónica Ciencia y Tecnología, 4(2), 166-214.

Petit, L. J. y Petit, D. R. (2003). Evaluating the importance of human-modified lands for neotropical bird conservation. Conservation Biology, 17(3), 687-694. https://doi.org/10.1046/j.1523-1739.2003.00124.x

Quiceno, N. J., Tangarife, G. M. y Álvarez, L. R. (2016). Estimación del contenido de biomasa, fijación de carbono y servicios ambientales, en un área de bosque primario en el resguardo indígena Piapoco Chigüiro-Chátare de Barrancominas, departamento 
del Guainía (Colombia). Luna Azul, 43, 171-202.

http://doi.org/10.17151/luaz.2016.43.9

Quintana, Y. G., Crespo, Y. A., Esponda, D. C. y Pérez,

Y. L. (2017). Contribución de herramientas ecológicas en la formación agropecuaria y forestal en las condiciones amazónicas del Ecuador. REDVET. Revista Electrónica de Veterinaria, 18(12), 1-11.

Ter Braak, C. J. (1986). Canonical correspondence analysis: a new eigenvector technique for multivariate direct gradient analysis. Ecology, 67(5), 1167-1179. https://doi.org/10.2307/1938672

Ter Braak, C. J. y Smilauer, P. (2012). Canoco reference manual and user's guide: software for ordination, version 5.0.

Ter Steege, H., Pitman, N. C., Sabatier, D., Baraloto, C., Salomão, R. P., Guevara, J. E., Phillips, O. L., Castilho, C. V., Magnusson, W. E. y Molino, J. F. (2013). Hyperdominance in the Amazonian tree flora. Science, 342(6156), 1243092.

Tobías-Baeza, A., Salvador-Morales, P., Sánchez-Hernández, R., Ruiz-Acosta, S.d.C. y Andrade-Prado, H. (2019). Composición florística y carbono en la vegetación arbórea de un área periurbana en Tabasco, México. Ecosistemas y Recursos Agropecuarios, 6(17), 369-376. http://doi.org/10.19136/era.a6n17.2009

Tong, X., Brandt, M., Yue, Y., Ciais, P., Jepsen, M. R., Penuelas, J., Wigneron, J. P., Xiao, X., Song, X. P. y Horion, S. (2020). Forest management in southern China generates short term extensive carbon sequestration. Nature Communications, 11(1), 1-10. https://doi.org/10.1038/s41467-019-13798-8

Torres, B., Vasseur, L., López, R., Lozano, P., García, Y., Arteaga, Y., Bravo, C., Barba, C. y García, A. (2019). Structure and above ground biomass along an elevation small-scale gradient: case study in an Evergreen Andean Amazon forest, Ecuador. Agroforestry Systems, 1-11. https://doi.org/10.1007/s10457-018-00342-8

Trugman, A. T., Medvigy, D., Hoffmann, W. A. y Pellegrini, A. F. (2018). Sensitivity of woody carbon stocks to bark investment strategy in Neotropical savannas and forests. Biogeosciences, 15(1). https://doi.org/10.5194/bg-15-233-2018

Ureta, A. M. (2015). Aporte de biomasa aérea de las especies arbóreas de la familia Myristicaceae en los bosques amazónicos del Perú. Revista de Biología Tropical, 63(1), 263-273.

Valdés-Sáenz, M. A., García-Quintana, Y., Escarré-Esteve, A., Flores, J., Geada-López, G., Arteaga-Crespo, Y. y Valdés-Sáenz, C. R. (2014). Estructura de un bosque natural perturbado de Pinus tropicalis Morelet en Galalón, Cuba. Botanical Sciences, 92(3), 417-423.

Vargas, E. P. R., Agudelo, E. D. S., Motta, A. Y. G., Delgado, P. A. M. y Valencia, W. H. (2019). Carbono almacenado en estrato arbóreo de sistemas ganaderos y naturales del municipio de Albania, Caquetá, Colombia. Revista Ciencia y Agricultura, 16(3), 35-46.

Vasco, C., Torres, B., Pacheco, P. y Griess, V. (2017). The socioeconomic determinants of legal and illegal smallholder logging: Evidence from the Ecuadorian Amazon. Forest Policy and Economics, 78, 133-140. https://doi.org/10.1016/j.forpol.2017.01.015

Vashum, K. T. y Jayakumar, S. (2012). Methods to estimate above-ground biomass and carbon stock in natural forests-a review. Journal of Ecosystem \& Ecography, 2(4), 1-7. http://doi.org/10.4172/2157-7625.1000116

Vega, R. R. (2020). Diversidad florística del bosque seco tropical en las subregiones bajo y medio Sinú, Córdoba, Colombia. Revista de Biología Tropical, 68(1). http://dx.doi.org/10.4172/2157-7625.1000116

Yepes, A., Herrera, J., Phillips, J., Cabrera, E., Galindo, G., Granados, E., Duque, Á., Barbosa, A., Olarte, C. y Cardona, M. (2015). Contribución de los bosques tropicales de montaña en el almacenamiento de carbono en Colombia. Revista de Biología Tropical, 63(1), 69-82. https://www.scielo.sa.cr/scielo.php?script=sci_arttext\&pid=S0034-77442015000100007

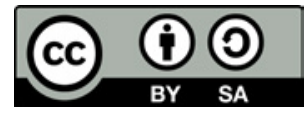

Colombia Forestal •ISSN 0120-0739 • e-ISSN 2256-201X • Bogotá-Colombia • Vol. 24 No. 1 • Enero-Junio de $2021 \bullet$ pp. 45-59 